\title{
Adjustment of superconductivity and ferromagnetism in the few-layered ferromagnet-superconductor nanostructures
}

\author{
Y.A. Izyumov ${ }^{1}$, M.G. Khusainov ${ }^{2,3,4}$, Y.N. Proshin ${ }^{2,3}$ \\ ${ }^{1}$ Institute of Metal Physics, Ural Division of RAS, Ekaterinburg 620219, Russia \\ ${ }^{2}$ Kazan State University, Kazan 420008, Russia \\ Email: Yurii.Proshin@ksu.ru \\ ${ }^{3}$ Max-Planck-Institute for the Physics of Complex Systems, Dresden 01871, Germany \\ ${ }^{4}$ «ostok» branch, Kazan State Tupolev Technical University, Chistopol' 422981, Russia
}

Received March 15, 2006, revised May 4, 2006

\begin{abstract}
The phase diagrams of the few-layered nanosystems consisting of dirty superconducting $(\mathrm{S})$ and ferromagnetic (F) metals are investigated within the framework of the modern theory of the proximity effect taking into account the boundary conditions. The $\mathrm{F} / \mathrm{S}$ tetralayer and pentalayer are shown to have considerably richer physics than the $\mathrm{F} / \mathrm{S}$ bi- and trilayer (due to the interplay between the 0 and $\pi$ phase superconductivity and the 0 and $\pi$ phase magnetism and nonequivalence of layers) and even the $\mathrm{F} / \mathrm{S}$ superlattices. It is proven that these systems can have different critical temperatures and fields for different $\mathrm{S}$ layers. This predicted decoupled superconductivity is found to manifest itself in its most striking way for $\mathrm{F} / \mathrm{S}$ tetralayer. It is shown that $\mathrm{F} / \mathrm{S} / \mathrm{F}^{\prime} / \mathrm{S}^{\prime}$ tetralayer is the most perspective candidate for use in superconducting spin nanoelectronics.
\end{abstract}

PACS: 74.78.Fk, 85.25.-j, 74.62.-c, 85.75.-d

Keywords: proximity effect, superconductivity, ferromagnetism, multilayers, critical temperature.

\section{Introduction}

The fabricated $\mathrm{F} / \mathrm{S}$ heterostructures consisting of alternating ferromagnetic metal (F) and superconducting $(\mathrm{S})$ layers are well explored systems (see the recent reviews [1-4] and references therein). Due to the proximity effect, the superconducting order parameter can be induced in the $\mathrm{F}$ layer; on the other hand, the neighboring pair of the F layers can interact with each other via the S layer. Such systems exhibit rich physics, which can be controlled by varying the thicknesses of the $\mathrm{F}$ and $\mathrm{S}$ layers, or by placing the $\mathrm{F} / \mathrm{S}$ structure in an external magnetic field. $\mathrm{Nu}-$ merous experiments on the $\mathrm{F} / \mathrm{S}$ structures (contacts, trilayers, and superlattices) have revealed nontrivial dependences of the superconducting transition temperature $T_{c}$ on the thickness of the ferromagnetic layer $d_{f}$.
Buzdin et al. [5] were first who formulated the boundary value problem for the pair amplitude (the Cooper pair wave function) in a dirty superconductor for the $\mathrm{F} / \mathrm{S}$ superlattice. The critical temperature $T_{c}$ that was also calculated as the $d_{f}$ function in Refs. 5, 6 exhibited both monotonic and nonmonotonic dependences. Oscillations of $T_{c}\left(d_{f}\right)$ were related to periodical switching of the ground superconducting state between the 0 and $\pi$ phases, so that the system chooses the state with higher transition temperature $T_{c}$. In the $\pi$ phase state the superconducting order parameter $\Delta$ in the neighboring $\mathrm{S}$ layers of the $\mathrm{F} / \mathrm{S}$ superlattice has the opposite sign, contrary to the 0 phase state in which $\Delta$ has same sign for all $\mathrm{S}$ layers. The experimental evidence of the $\pi$ superconducting state in the $\mathrm{F} / \mathrm{S}$ systems has been discussed in the review [2]. The concept of a $\pi$ junction was first proposed by Bulaevsky et al. [7]. 
In subsequent studies [8-11] the boundary conditions have been derived from the microscopic theory, and they are valid for arbitrary transparency of the $\mathrm{F} / \mathrm{S}$ interface. The solution of the boundary value problem [8-15] has revealed an additional mechanism of nonmonotonic dependence of $T_{c}$ due to modulation of the pair amplitude flux from the $\mathrm{S}$ layer to the $\mathrm{F}$ layer. This modulation is caused by the change of the FM layer thickness $d_{f}$. Moreover, it has also resulted in a prediction of different types of behavior $T_{c}\left(d_{f}\right)$ such as reentrant $[8-10,16]$ and periodically reentrant superconductivity [8-10]. Note that both the oscillations and the reentrant behavior of $T_{c}\left(d_{f}\right)$ can appear not only in the $\mathrm{F} / \mathrm{S}$ superlattice but also in simple $\mathrm{F} / \mathrm{S}$ bilayer and $\mathrm{F} / \mathrm{S} / \mathrm{F}$ trilayer systems in which the $\pi$ phase superconductivity is impossible in principle! The reentrant character of superconductivity that we have predicted has been recently observed experimentally in the $\mathrm{Fe} / \mathrm{V} / \mathrm{Fe}$ trilayer [17].

Now it may be considered as proven [1] that superconductivity in the layered $\mathrm{F} / \mathrm{S}$ systems is a combination of the BCS pairing with a zero total momentum of the pairs in the $\mathrm{S}$ layers and the pairing due to the Larkin - Ovchinnikov-Fulde-Ferrell (LOFF) mechanism $[18,19]$ with a nonzero three-dimensional (3D) momentum of the pairs $\mathbf{k}$ in the $\mathrm{F}$ layer. The LOFF pairs momentum $k \simeq 2 I / v_{f}$ is determined by the Fermi surface splitting caused by the internal exchange field $I$ (where $v_{f}$ is the Fermi velocity in the $\mathrm{F}$ layers). Usually it is assumed [5,6,8-15,20,21] that the momentum of the LOFF pairs is directed across the $\mathrm{F} / \mathrm{S}$ interface (the so-called one-dimensional (1D) case).

Of special interest is the study of the multilayered $\mathrm{F} / \mathrm{S}$ structures, in which various types of magnetic order can arise in the $\mathrm{F}$ layers due to their indirect interaction via the S layers. Recently the theory of the proximity effect has been developed for the $\mathrm{F} / \mathrm{S}$ structures taking into account the inverse influence of superconductivity on magnetism of the $\mathrm{F}$ layers and on mutual orientation of their magnetizations. This aspect of the proximity effect has been studied for the $\mathrm{F} / \mathrm{S} / \mathrm{F}$ trilayer «spin-switch» $[22,23]$, and in the $\mathrm{F} / \mathrm{S}$ bilayer at exploring the possibility of the cryptoferromagnetic state [24,25] and possible magnetic correlations acquired by a superconductor at the $\mathrm{S} / \mathrm{F}$ interface [26]. The long-range proximity effect due to triplet superconductivity that arises in the case of non-collinear alignment of magnetizations in the $\mathrm{F}$ layers has been studied for the $\mathrm{F} / \mathrm{S} / \mathrm{F}$ trilayer system in Refs. 27-30.

The multilayered $\mathrm{F} / \mathrm{S}$ systems have additional competition between the 0 and $\pi$ phase types of superconductivity. Along to an interplay between the 0 and $\pi$ phase magnetism this leads to a new classification of the $\mathrm{F} / \mathrm{S}$ superlattice states $[1,31,32]$. In this classification scheme the four different states $(\varphi \chi)$ are distinguished by the phases of the superconducting (the first symbol, $\varphi$ ) and magnetic (the second one, $\chi$ ) order parameters. So, the $\mathrm{F} / \mathrm{S}$ superlattice possesses two ferromagnetic superconducting (FMS) states (00, $\pi 0)$, and two antiferromagnetic superconducting (AFMS) ones $(0 \pi$ and $\pi \pi)$. In the AFMS states the phases $\chi$ in the neighboring $F$ layers are shifted by $\pi$, i.e. the exchange fields $I$ have opposite signs in the neighboring $\mathrm{F}$ layers. This state with antiparallel alignment of the corresponding magnetizations may be considered as a manifestation of the $\pi$ phase magnetism. Similar to the $\mathrm{F} / \mathrm{S} / \mathrm{F}$ trilayer $[22,23]$, in the case of the $\mathrm{F} / \mathrm{S}$ superlattice the AFM ordering of the magnetizations of all $\mathrm{F}$ layers leads to the significant reduce of the pair-breaking effect of the exchange field $I$ for the $\mathrm{S}$ layers, and to the raise of the critical temperature of the layered system. This theoretical prediction has been experimentally confirmed for the $\mathrm{Gd} /$ La superlattices [33]. Goff et al. have observed that the superlattices with prepared antiferromagnetic ordering of the magnetizations in the adjacent Gd layers undergo the transition into a superconducting state at considerably higher temperatures in comparison with the samples with ferromagnetic ordering magnetization of the Gd layers. This mutual accommodation between the superconducting and magnetic order parameters reflects a quantum coupling between the boundaries.

The $\mathrm{F} / \mathrm{S}$ nanostructures possess two data-recording channels: the superconducting one determined by conducting properties of the $\mathrm{S}$ layers, and the magnetic one determined by ordering of the $\mathrm{F}$ layer magnetizations. The $\mathrm{F} / \mathrm{S} / \mathrm{F}$ trilayer devices, proposed in Refs. 22-24, operate through transitions between two states (the superconducting (S) and normal $(\mathrm{N})$ ones) that are induced by changes of the mutual ordering of the magnetizations of the adjacent $\mathrm{F}$ layers. These changes are controlled by an external magnetic field $H$. The data stored in the superconducting and magnetic channels of this switch device change simultaneously, and the magnetic order completely determines the «superconducting information». The scheme of a complex «spin device» with five possible states on the basis of the $\mathrm{F} / \mathrm{S}$ superlattices, in which the superconducting and magnetic data-recording channels can be controlled separately, has been proposed in Refs. 31, 32.

However, both from the point of view of manufacturing and the «layer-by-layer» control by a weak magnetic field, the few-layered systems with a limited number of layers are more interesting objects. So, in 
Sec. 2 we theoretically investigate both well explored $\mathrm{F} / \mathrm{S}$ systems (bilayer, trilayers, and superlattice) and scantily-known $\mathrm{F} / \mathrm{S}$ tetra- and pentalayer (see Fig. 1). We solve the Usadel equations for these structures taking into account the boundary conditions and possible competition between the 0 and $\pi$ phase magnetism and the 0 and $\pi$ phase superconductivity. In Sec. 3 we construct the phase diagrams with an optimal set of parameters and discuss possible difference of critical temperatures of different $\mathrm{S}$ layers.

\section{Few-layered $F / S$ structures}

Some layered $\mathrm{F} / \mathrm{S}$ structures have been studied extensively in recent years both experimentally and theoretically. Mainly the $\mathrm{F} / \mathrm{S}$ bilayers (Fig. 1,a), the $\mathrm{F} / \mathrm{S} / \mathrm{F}($ Fig. 1,b), S $/ \mathrm{F} / \mathrm{S}$ (Fig. 1,c) trilayers, and $\mathrm{F} / \mathrm{S}$ superlattices were explored. The corresponding references could find in the recent reviews $[1,3,4]$. In this paper we consider both these systems and poorly explored few-layered $\mathrm{F} / \mathrm{S}$ systems: the $\mathrm{F} / \mathrm{S} / \mathrm{F}^{\prime} / \mathrm{S}^{\prime}$ tetralayer (Fig. 1,d) and the $\mathrm{F} / \mathrm{S} / \mathrm{F}^{\prime} / \mathrm{S}^{\prime} / \mathrm{F}^{\prime \prime}$ pentalayer (Fig. 1,e) with specific boundary conditions.

The proposed geometry of few-layered systems (see Fig. 1) would allow us to simplify the solutions, and, thereafter, to compare the obtained results for the tetralayer and pentalayer structures with the ones for the bilayer, trilayers and superlattice cases. The primes indicate possible difference in properties of layers made of identical material.
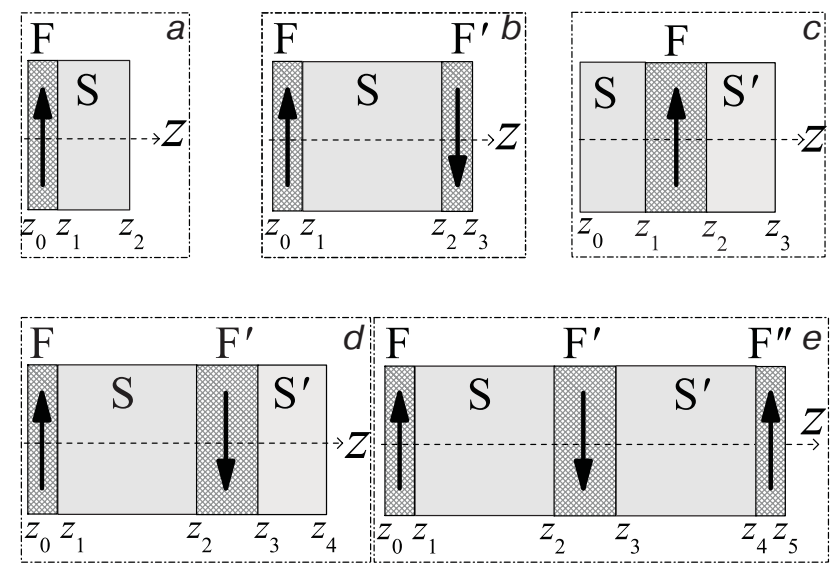

Fig. 1. The geometry of various few-layered systems. Bi-layer $\mathrm{F} / \mathrm{S}(a)$, two trilayers $\mathrm{F} / \mathrm{S} / \mathrm{F}^{\prime}(b)$ and $\mathrm{S} / \mathrm{F} / \mathrm{S}^{\prime}$ (c), tetralayer $\mathrm{F} / \mathrm{S} / \mathrm{F}^{\prime} / \mathrm{S}^{\prime} \quad(d)$, and pentalayer $\mathrm{F} / \mathrm{S} / \mathrm{F}^{\prime} / \mathrm{S}^{\prime} / \mathrm{F}^{\prime \prime}(e)$ are shown. Vertical arrows show the directions (in-line) of the magnetizations that play the role of the magnetic order parameter. Here $z_{1}=0$, and thicknesses of outer $\mathrm{F}$ layers and $\mathrm{S}$ layers are equal to $d_{f} / 2$ and $d_{s} / 2$, where $d_{f}$ and $d_{s}$ are thicknesses of inner $\mathrm{F}$ and $\mathrm{S}$ layers, correspondingly.
To find the critical temperature we assume the usual relation between the energsy parameters of the system $\varepsilon_{F} \gg 2 I \gg T_{C S}$, where $\varepsilon_{F}$ is the Fermi energy and $T_{c S}$ is the critical temperature of the $\mathrm{S}$ material. We also suppose the dirty limit conditions $l_{s} \ll<\xi_{s} \ll<\xi_{s 0}, l_{f} \ll<a_{f} \ll<\xi_{f}$. Here $l_{s, f}=v_{s, f} \tau_{s, f}$ is the mean free path length for the $\mathrm{S}(\mathrm{F})$ layer; $v_{s, f}$ is the Fermi velocity; $\xi_{s, f}=\left(D_{s, f} / 2 \pi T_{c s}\right)^{1 / 2}$ is the superconducting coherence length; $a_{f}=v_{f} / 2 I$ is the spin stiffness length; $\xi_{s 0}$ is the BCS coherence length; $D_{s, f}=v_{s, f} l_{s, f} / 3$ is the diffusion coefficient. For simplicity we will use the $1 \mathrm{D}$ model when both order parameters and the pair amplitude depend only on $z$ $[1,21]$.

In this case, the common boundary value problem [10] for each layer is reduced to the Gor'kov self-consistency equations for the Gor'kov function $F(z, \omega)$ (note, in this paper we often use the term «pair amplitude» for $F(z, \infty)$ due to traditions [1] and for simplicity)

$$
\begin{aligned}
& \Delta_{s}(z)=2 \lambda_{s} \pi T \operatorname{Re} \sum_{\omega>0}^{\prime} F_{s}(z, \omega), \\
& \Delta_{f}(z)=2 \lambda_{f} \pi T \operatorname{Re} \sum_{\omega>0}^{\prime} F_{f}(z, \omega) .
\end{aligned}
$$

and to the Usadel equations, that appear for the $\mathrm{S}$ and $\mathrm{F}$ layers as follows

$$
\begin{aligned}
& {\left[\omega-\frac{D_{s}}{2} \frac{\partial^{2}}{\partial^{2} z}\right] F_{S}(z, \omega)=\Delta_{s}(z),} \\
& {\left[\omega+i I-\frac{D_{f}}{2} \frac{\partial^{2}}{\partial^{2} z}\right] F_{f}(z, \omega)=\Delta_{f}(z) .}
\end{aligned}
$$

In Eqs. (1), (2) $0=\pi T(2 n+1)$ is the Matsubara frequency; $\Delta_{s(f)}$ and $\lambda_{s(f)}$ are the superconducting order parameter and the electron-electron coupling constant in the $\mathrm{S}(\mathrm{F})$ layers, respectively. The prime on the summation sign indicates cutoff at the Debye frequency ${ }^{\omega_{D}} D$. The diffusion coefficient $D_{f}$ in the $\mathrm{F}$ layer is assumed to be real rather than complex [1] since the difference between its two values is insignificant under the conditions $2 I \tau_{f}<<1$ used below (see discussion in Ref. 21).

The coupling between the superconducting and ferromagnetic layers is provided by corresponding boundary conditions, which connect the pair amplitude fluxes with the pair amplitude jumps on the interfaces of the layers, and are written in the following form [8-10] 


$$
\begin{aligned}
& \left.\frac{4}{\sigma_{s} v_{s}} D_{s} \frac{\partial F_{s}(z, \omega)}{\partial z}\right|_{z=z_{i}}=\left.\frac{4}{\sigma_{f} v_{f}} D_{f} \frac{\partial F_{f}(z, \omega)}{\partial z}\right|_{z=z_{i}}= \\
& = \pm\left[F_{s}\left(z_{i} \pm 0, \omega\right)-F_{f}\left(z_{i} \mp 0, \omega\right)\right] .
\end{aligned}
$$

Here index $i$ numbers the inner interfaces. The upper signs are chosen at the $\mathrm{F} / \mathrm{S}$ boundaries, the lower ones are chosen at the $\mathrm{S} / \mathrm{F}$ boundaries. The pair amplitude fluxes through the outside boundaries $\left(z_{\text {left }}=z_{0}\right.$ and $z_{\text {right }}$, see Fig. 1$)$ are absent

$$
\left.\frac{\partial F(z, \omega)}{\partial z}\right|_{z=z_{\text {left }}}=\left.\frac{\partial F(z, \omega)}{\partial z}\right|_{z_{\text {right }}}=0 .
$$

In Eq. (3) $\sigma_{s(f)}$ is the boundary transparency at the $\mathrm{S}(\mathrm{F})$ side $\left(0 \leq \sigma_{s, f}<\infty\right)[1,10]$. They satisfy the detailed balance condition: $\sigma_{s} v_{s} N_{s}=\sigma_{f} v_{f} N_{f}$, where $N_{s(f)}$ is the density of states at the Fermi level.

The last conditions (3b) distinguish the case of few-layered structures (Fig. 1) from the $\mathrm{F} / \mathrm{S}$ superlattice case $[1,35]$ in which the pair amplitude $F$ should satisfy to periodical conditions $F(z+L, I)=\mathrm{e}^{i \varphi} F\left(z, I \mathrm{e}^{i \chi}\right)$, where $L=d_{s}+d_{f}$ is the superlattice period, and $\varphi$ and $\chi$ are the phases of superconducting and magnetic order parameters, respectively.

In order to calculate the critical temperatures of this $\mathrm{F} / \mathrm{S}$ system taking into consideration the boundary transparencies, thicknesses of layers, etc., we should solve the system of equations (2) and (3) together with the self-consistency equations (1).

The powerful pair-breaking action of the exchange field $I\left(I \gg \pi T_{C S}\right)$ is the basic mechanism for the destruction of superconductivity in the $\mathrm{F} / \mathrm{S}$ systems. For simplicity [10] assume that $\lambda_{f}=0\left(\Delta_{f}=0\right)$ in the $F$ layers. We will search the solutions of equations (1)-(3) for the inner layers as a linear combination of symmetric and antisymmetric functions relative to the centers of the corresponding S and F layers. These pair amplitudes (Gor'kov functions) look the same as in the superlattice case [32]. The zero flux of the pair amplitude through the outside boundaries (3b) determines only the even cosine-like functions for the outer layers. At these boundaries the antinodes should be fixed.

Since we are mainly interested in performing qualitative studies of the properties of the few-layered nanostructures, we will use the single-mode approximation to obtain the analytical solution of the complicated boundary value problem. However, when quantitative estimates are needed (to fit theoretical results to experimental data) the latter approximation works well only for a certain range of the values of the parameters in the problem $[13,14]$. According to our estimates [10] the optimal set of parameters used below is close to this range $\left(d_{s(f)}>\xi_{s(f)}\right)$. Note, that in any approximation (single-mode, multi-mode, etc. [14]) the symmetry of the problem determines the possible solutions.

\subsection{Simple few-layered systems ( ${ }^{i}$ - and tri-layers) and superlattice}

Firstly let us consider the well-explored $\mathrm{F} / \mathrm{S}$ bilayer and two types of trilayers. The simplest solutions of the boundary value problem (2), (3) for the $\mathrm{F} / \mathrm{S}$ bilayer (Fig. 1,a) can be written the following form [1]

$$
\begin{aligned}
& F_{f}=B \frac{\cos k_{f}\left(z+d_{f} / 2\right)}{\cos \left(k_{f} d_{f} / 2\right)}, \quad-d_{f} / 2<z<0, \\
& F_{s}=A \cos \frac{k_{s}\left(z-d_{s} / 2\right)}{\cos \left(k_{s} d_{s} / 2\right)}, \quad 0<z<d_{s} / 2 .
\end{aligned}
$$

Here and below $k_{s}(f)$ are the components of the wave vector that describe spatial changes of the pair amplitudes $F_{s(f)}$ across the layers (along the $z$ axis) independently of the frequency $\omega$. The chosen form of the pair amplitudes is related to the symmetry of bilayer and corresponding boundary conditions (3).

The complex values of wave vector $k_{f}$ for the ferromagnetic layer is defined as [1,21]

$$
k_{f}^{2}=-\frac{2 i I}{D_{f}} .
$$

Substituting the solutions (4) into the self-consistency equations (1) and performing the standard summation over $\omega$ we derive the usual Abrikosov-Gor'kov type equation for the reduced superconducting transition temperatures $t_{c}$ of the $\mathrm{S}$ layer

$$
\ln t_{c}=\Psi\left(\frac{1}{2}\right)-\operatorname{Re} \Psi\left(\frac{1}{2}+\frac{D_{s} k_{s}^{2}}{4 \pi T_{c s} t_{c}}\right)
$$

where $t_{c}=T_{c} / T_{C S} ; \Psi(x)$ is the digamma function, and the pair-breaking parameter $D_{s} k_{s}^{2}$ is the solution of the other transcendental equation, that is determined from the condition of nontrivial compatibility of set of linear equations for factors $B, A$. In turn this set can be obtained by substitution of the solutions (4) in (3). In this way we obtain one possible state for the $\mathrm{F} / \mathrm{S}$ bilayer case

$$
\alpha \gamma+1=0
$$

where

$$
\alpha=\frac{4 D_{s} k_{s}}{\sigma_{s} v_{s}} \tan \frac{k_{s} d_{s}}{2}-1, \quad \gamma=-\frac{4 D_{f} k_{f}}{\sigma_{f} v_{f}} \tan \frac{k_{f} d_{f}}{2}+1 .
$$


Equation (7) is the sole equation on $k_{S}$ which does not depend on the orientation of magnetization in the F layer. This expression (7) can be rewritten in long-known form [1]

$$
D_{s} k_{s}^{00} \tan \frac{k_{s}^{00} d_{s}}{2}=\frac{\sigma_{s} v_{s}}{4-\left(\frac{\sigma_{s} v_{f}}{D_{f} k_{f}}\right) \cot \frac{k_{f} d_{f}}{2}} .
$$

Found from Eq. (7) or (9) the $k_{s}$ wave vector being substituted to (6) allow us to calculate the $T_{c}$ of bilayer. It leads to the well known 00 type of solution (according classification [1] the 00 state is one with the 0 phase superconductivity and the 0 phase magnetism).

For the $\mathrm{F} / \mathrm{S} / \mathrm{F}^{\prime}$ trilayer we should change second expression in Eq. (4) for the inner $\mathrm{S}$ layer, adding the sin-like summand, and add new expression for the outer $\mathrm{F}^{\prime}$ layer. Thus, three functions are

$$
\begin{aligned}
& F_{f}=B \frac{\cos k_{f}\left(z+d_{f} / 2\right)}{\cos \left(k_{f} d_{f} / 2\right)},-d_{f} / 2<z<0, \\
& F_{s}=A \frac{\cos k_{s}\left(z-d_{s} / 2\right)}{\cos \left(k_{s} d_{s} / 2\right)}+C \frac{\sin k_{s}\left(z-d_{s} / 2\right)}{\sin \left(k_{s} d_{s} / 2\right)},(10 \mathrm{~b}) \\
& 0<z<d_{s}, \\
& F_{f}^{\prime}=B^{\prime} \frac{\cos k_{f}^{\prime}\left(z-d_{s}-d_{f} / 2\right)}{\cos \left(k_{f}^{\prime} d_{f} / 2\right)}, d_{s}<z<d_{s}+d_{f} / 2 .
\end{aligned}
$$

Recall, that in the paper the quantities related to the different $\mathrm{S}$ and $\mathrm{F}$ layers layer are denoted by the primes in correspondence with Fig. 1. In Eq. (10b) the first term is responsible for the symmetric 0 phase solution, while the second term is responsible for the appearance of antisymmetric $\pi$ phase solution.

Without loss in generality we assume that the magnetization in the left outer $\mathrm{F}$ layer is always upward in all considered $\mathrm{F} / \mathrm{S}$ systems (Fig. 1). Then, in the framework of the made approximations, the complex values of wave vectors $k_{f}$ and $k_{f}^{\prime}$ for the $\mathrm{F}$ and $\mathrm{F}^{\prime}$ layers, correspondingly, with the upward magnetizations are defined as $[1,21]$

$$
k_{f}^{2}=\left(k_{f}^{\prime}\right)^{2}=-\frac{2 i I}{D_{f}} .
$$

Thus, the Eqs. (11a) are valid for the case of the mutual ferromagnetic (FM) ordering of the magnetizations in all ferromagnetic layers. When we have antiferromagnetic (AFM) configuration with magnetization $\mathbf{M}^{\prime}$ oriented «downward» $\left(I^{\prime}=-I\right.$, i.e. the phase $\chi$ of the magnetic order parameter equals $\pi$ ), the value of $k_{f}^{\prime}$ for the $F^{\prime}$ layer can be found from following expression

$$
\left(k_{f}^{\prime}\right)^{2}=\frac{2 i I}{D_{f}}=\left(k_{f}^{2}\right)^{*},
$$

where $k_{f}$ is defined by Eq. (11a).

From condition of nontrivial compatibility of set of linear equations for factors $B, A, C, B^{\prime}$ we obtain two different solutions. One of them is the 00 state for FM ordering of magnetizations of the adjacent $F$ and $F^{\prime}$ layers, which coincides with above stated solution for the $\mathrm{F} / \mathrm{S}$ bilayer (7). Another one is the $0 \pi$ state which corresponds the 0 phase type of superconductivity and $\pi$ phase type of magnetism (i.e., AFM ordering of magnetizations of the adjacent $\mathrm{F}$ and $\mathrm{F}^{\prime}$ layers)

$$
\alpha \beta|\gamma|^{2}+(\beta-\alpha) \operatorname{Re} \gamma=1,
$$

when wave vector $k_{f}^{\prime}$ in the $\mathrm{F}^{\prime}$ layer is complex conjugate to $k_{f}$ in the $\mathrm{F}$ layer (see Eq. (11b) above). In Eq. (12) we use this fact, that leads to $\gamma^{\prime}=\gamma^{*}$, and introduce denotation

$$
\beta=\frac{4 D_{s} k_{s}}{\sigma_{s} v_{s}} \cot \frac{k_{s} d_{s}}{2}+1
$$

The critical temperatures corresponding to both states can be calculated with Eq. (6) after substitution of $k_{s}^{00}$ found from (8) and $k_{s}^{0 \pi}$ found from (12).

To obtain the set of solutions for the $\mathrm{S} / \mathrm{F} / \mathrm{S}^{\prime}$ trilayers (Fig. 1,c) we have to simply exchange the indices and factors in Eqs. (10): $s \leftrightarrow f$; and $B \rightarrow A$, $B^{\prime} \rightarrow A^{\prime}, A \rightarrow B$, and $C \rightarrow D$.

The states of the $\mathrm{S} / \mathrm{F} / \mathrm{S}^{\prime}$ trilayer are independent of orientation of magnetization in the $\mathrm{F}$ layer, it corresponds to the 0 phase of magnetic order parameter. In this case we formally have two equations for critical temperatures for layer S (see Eq. (6)) and for layer $S^{\prime}$ (to get an equation for $t_{c}^{\prime}$ it is necessary to exchange $t_{c}$ for $t_{c}^{\prime}=T_{c}^{\prime} / T_{c s}$ and $k_{s}$ for $k_{s}^{\prime}$ in Eq. (6)). The critical temperatures depend heavily on the relative sign of the superconducting order parameter $\Delta$ in the $S$ and $S^{\prime}$ layers. So, the two known states are obtained. The first one is the 00 sate defined by Eq. (8) which is identical for both $\mathrm{S}$ and $\mathrm{S}^{\prime}$ layers (hence $\alpha^{\prime}=\alpha$, then $\left.T_{c}^{\prime}=T_{c}\right)$. The second solution is the $\pi 0$ state

$$
\alpha \delta+1=0 ; \leftarrow \text { layer } \mathrm{S}, \alpha^{\prime} \delta+1=0 ; \leftarrow \text { layer } \mathrm{S}^{\prime},
$$

where denotation $\delta$ is

$$
\delta=\frac{4 D_{f} k_{f}}{\sigma_{f} v_{f}} \cot \frac{k_{f} d_{f}}{2}+1 .
$$

The Eqs. (14) also lead to the $\alpha^{\prime}=\alpha$, then $k_{s}^{\prime}=k_{s}$, and hence we also have common critical temperature in the $\pi 0$ state for whole sample, i.e. $T_{c}^{\prime}=T_{c}$. Note, the wave vector $k_{S}$ in the $\pi 0$ state can be found from Eq. (9) by substituting $k_{s}^{\pi 0}$ for $k_{s}^{00}$ in the left-hand 
side and $-\tan \left(k_{f} d_{f} / 2\right)$ for $\cot \left(k_{f} d_{f} / 2\right)$ in its right-hand side.

Before a consideration of the $\mathrm{F} / \mathrm{S}$ tetralayer and pentalayer we shortly discuss the $\mathrm{F} / \mathrm{S}$ superlattice case, in which the full competition between the 0 and $\pi$ phase types of superconductivity and magnetism is possible $[1,31,32,35]$. The pair amplitudes for all $\mathrm{S}$ layers will be analogous to one for inner $\mathrm{S}$ layer in the $\mathrm{F} / \mathrm{S} / \mathrm{F}$ trilayer $(10 \mathrm{~b})$, the ones for all $\mathrm{F}$ layers will correspond to one for inner $\mathrm{F}$ layer in the $\mathrm{S} / \mathrm{F} / \mathrm{S}$ trilayer. Following to the cited papers the superconducting states of the $\mathrm{F} / \mathrm{S}$ superlattice can be classified using four different sets $\varphi \chi: 00,0 \pi, \pi 0$, and $\pi \pi$. Corresponding wave vectors $k_{s}$ for three of these states are already defined by Eqs. (7), (12), (14), correspondingly. The last $\pi \pi$ state is determined by $k_{S}$, which is found by following equation $[1,31,32]$

$$
\alpha \beta|\delta|^{2}+(\beta-\alpha) \operatorname{Re} \delta=1 .
$$

Note, that the competition between the 0 phase superconductivity generated by Eq. (7) and the $\pi$ phase superconductivity generated by Eq. (14) for the $\mathrm{F} / \mathrm{S}$ superlattice was firstly proposed fifteen years ago $[5,6]$ to explain nonmonotone experimental $T_{c}\left(d_{f}\right)$ dependence. A manifestation of $\pi$ phase superconductivity in the experiments on the Josephson current can be found in the review [2].

\subsection{Tetra- and penta-layers}

For the $\mathrm{F} / \mathrm{S} / \mathrm{F}^{\prime} / \mathrm{S}^{\prime}$ tetralayer (see Fig. 1,d) we have the following form of solutions

$$
\begin{aligned}
& F_{f}=B \frac{\cos k_{f}\left(z+d_{f} / 2\right)}{\cos \left(k_{f} d_{f} / 2\right)},-d_{f} / 2<z<0, \\
& F_{s}=A \frac{\cos k_{s}\left(z-d_{s} / 2\right)}{\cos \left(k_{s} d_{s} / 2\right)}+C \frac{\sin k_{s}\left(z-d_{s} / 2\right)}{\sin \left(k_{s} d_{s} / 2\right)} \\
& 0<z<d_{s} \\
& F_{f}^{\prime}=B^{\prime} \frac{\cos k_{f}^{\prime}\left(z-d_{s}-d_{f} / 2\right)}{\cos \left(k_{f}^{\prime} d_{f} / 2\right)}+ \\
& +D^{\prime} \frac{\sin k_{f}^{\prime}\left(z-d_{s}-d_{f} / 2\right)}{\sin \left(k_{f}^{\prime} d_{f} / 2\right)}, d_{s}<z<\left(d_{f}+d_{s}\right), \\
& F_{s}^{\prime}=A^{\prime} \frac{\cos k_{s}^{\prime}\left(z-d_{f}-3 d_{s} / 2\right)}{\cos \left(k_{s}^{\prime} d_{s} / 2\right)} \\
& \left(d_{f}+d_{s}\right)<z<\left(d_{f}+3 d_{s} / 2\right)
\end{aligned}
$$

At last for the $\mathrm{F} / \mathrm{S} / \mathrm{F}^{\prime} / \mathrm{S}^{\prime} / \mathrm{F}^{\prime \prime}$ pentalayer (see Fig. 1,e) in comparison with Eqs. (17) we should re- place the equation (17d) on the equation (18a) and add the equation (18b)

$$
\begin{aligned}
& F_{s}{ }^{\prime}=A^{\prime} \frac{\cos k_{s}^{\prime}\left(z-d_{f}-3 d_{s} / 2\right)}{\cos \left(k^{\prime}{ }_{s} d_{s} / 2\right)}+ \\
& +C^{\prime} \frac{\sin k_{s}^{\prime}\left(z-d_{f}-3 d_{s} / 2\right)}{\sin \left(k^{\prime}{ }_{s} d_{s} / 2\right)}, \\
& d_{f}+d_{s}<z<d_{f}+2 d_{s} \\
& F_{f}^{\prime \prime}=B^{\prime \prime} \frac{\cos k_{f}^{\prime \prime}\left(z-3 d_{f} / 2-2 d_{s}\right)}{\cos \left(k_{f}^{\prime \prime} d_{f} / 2\right)}, \\
& d_{f}+2 d_{s}<z<3 d_{f} / 2+2 d_{s} .
\end{aligned}
$$

So the full set of solutions for the considered pentalayer includes Eqs. (17a), (17b), (17c), (18a), (18b). Recall that according to our denotations all quantities related to the $\mathrm{F}^{\prime \prime}$ layer are denoted by the double primes. Once again, for convenience we assume that the magnetization in the left $\mathrm{F}$ layer is always upward (Fig. 1).

Naturally, by symmetry, for the bilayer, both trilayers and superlattice, studied before (see Refs. 1, 2, 4 and references therein) all superconducting layers are placed in identical surroundings. The same we can say about symmetrical configurations of the $\mathrm{F} / \mathrm{S} / \mathrm{F}^{\prime} / \mathrm{S}^{\prime} / \mathrm{F}^{\prime \prime}$ pentalayer. In the end this leads to the identical superconducting properties of all the $\mathrm{S}$ layers, that is, all $\mathrm{S}$ layers have common critical temperature. In contrary, for the $\mathrm{F} / \mathrm{S} / \mathrm{F}^{\prime} / \mathrm{S}^{\prime}$ tetralayer and $\mathrm{F} / \mathrm{S} / \mathrm{F}^{\prime} / \mathrm{S}^{\prime} / \mathrm{F}^{\prime \prime}$ pentalayer (asymmetrical case) the solutions will be different for the different $\mathrm{S}$ and $\mathrm{S}^{\prime}$ ( $\mathrm{F}$ and $\mathrm{F}^{\prime}$ ) layers. This fact reflects the general property of these systems: the nonequivalence of layers of the same type, which results in different superconducting properties of the $\mathrm{S}$ and $\mathrm{S}^{\prime}$ layers.

For the $\mathrm{F} / \mathrm{S} / \mathrm{F}^{\prime} / \mathrm{S}^{\prime}$ tetralayer the nonequivalence of the $\mathrm{S}(\mathrm{F})$ and $\mathrm{S}^{\prime}\left(\mathrm{F}^{\prime}\right)$ layers are essential. In common case they have different local surroundings. The wave vectors $k_{S}$ for the $\mathrm{S}$ layer and $k_{S}^{\prime}$ for the $\mathrm{S}^{\prime}$ layer are determined from the condition of nontrivial compatibility of the corresponding set of equations on the factors $B, A, C, B^{\prime}, D^{\prime}, A^{\prime}$, which can be obtained after substitution of the expressions for pair amplitudes (17) into Eqs. (3). It is possible to factorize the corresponding determinant and to obtain the following equation

$$
\begin{aligned}
& \left(\alpha^{\prime} \delta^{\prime}+1\right)\left[(\alpha \gamma+1)\left(\beta \gamma^{\prime}-1\right)+(\beta \gamma-1)\left(\alpha \gamma^{\prime}+1\right)\right]+ \\
& +\left(\alpha^{\prime} \gamma^{\prime}+1\right)\left[(\alpha \gamma+1)\left(\beta \delta^{\prime}-1\right)+(\beta \gamma-1)\left(\alpha \delta^{\prime}+1\right)\right]=0 .
\end{aligned}
$$

Equation (19) can be simplified by taking into account the independence of the solutions for the $\mathrm{S}$ and $\mathrm{S}^{\prime}$ layers and knowing the solutions for the superlattice case. It is possible to obtain the following 
sets of equations on $k_{S}$ and $k_{S}^{\prime}$, which are different for the FM and AFM configurations. Note that only equations leading to the finite nonzero critical temperature are kept in these sets (see Eqs. (20) and (21) below).

For the FM ordering of the magnetizations we obtain two cases FM $\left(a, a^{\prime}\right)$ and FM $\left(b, b^{\prime}\right)$

$\mathrm{FM}\left(\begin{array}{c}a \\ a^{\prime}\end{array}\right) \rightarrow\left\{\begin{array}{l}\alpha \gamma+1=0 ; \leftarrow \text { layer } \mathrm{S} \rightarrow(00) \\ \alpha^{\prime} \gamma+1=0 ; \leftarrow \text { layer } \mathrm{S}^{\prime} \rightarrow(00)^{\prime}\end{array}\right.$
$\mathrm{FM}\left(\begin{array}{c}b \\ b^{\prime}\end{array}\right) \rightarrow\left\{\begin{array}{l}2 \alpha \gamma \beta \delta+(\beta-\alpha)(\gamma+\delta)=2 ; \leftarrow \text { layer } \mathrm{S} \rightarrow(\tilde{\pi} 0) \\ \alpha^{\prime} \delta+1=0 ; \leftarrow \text { layer } \mathrm{S}^{\prime} \rightarrow(\pi 0)^{\prime} .\end{array}\right.$

Here $\gamma^{\prime}$ and $\delta^{\prime}$ for the $F^{\prime}$ layer are substituted by $\gamma$ and $\delta$, respectively, due to Eq. (11a). We see that cases $\mathrm{FM}(a)$ and $\mathrm{FM}\left(a^{\prime}\right)$ coincide with known 00 solutions for layers $\mathrm{S}$ and $\mathrm{S}^{\prime}$, correspondingly. The $\mathrm{FM}\left(b^{\prime}\right)$ one for the outer $\mathrm{S}^{\prime}$ layer is the $\pi 0$ solution also known for the $\mathrm{S} / \mathrm{F} / \mathrm{S}$ trilayer (14) and superlattice case. The $\mathrm{FM}(b)$ for the inner S layer is completely new solution. Its presence is related to the external boundary conditions (3b) since the pair amplitudes (17a), (17d) contain only even cosine solutions in contrary to the $\mathrm{F} / \mathrm{S}$ superlattice case. This state is the new $\pi$ superconducting state, and in order to distinguish it from the previous superlattice solutions we will denote the new ones with tilde $\tilde{\pi}(\chi=0, \pi$ is the phase of the magnetic order parameter). Thus the FM (b) state can be denoted as the $\tilde{\pi 0}$ state of the tetralayer.

According to equation (11b), when $k_{f}^{\prime}=k_{f}^{*}$ (leading to $\gamma^{\prime}=\gamma^{*}$ and $\delta^{\prime}=\delta^{*}$ ), for the AFM ordering of magnetizations in the tetralayer we have two other $\pi$ magnetic cases $\operatorname{AFM}\left(c, c^{\prime}\right)$ and $\operatorname{AFM}\left(d, d^{\prime}\right)$

$\operatorname{AFM}\left(\begin{array}{l}c \\ c^{\prime}\end{array}\right) \rightarrow\left\{\begin{array}{l}\alpha \beta|\gamma|^{2}+(\beta-\alpha) \operatorname{Re} \gamma=1 ; \leftarrow \text { layer } \mathrm{S} \rightarrow(0 \pi) \\ \alpha^{\prime} \gamma^{*}+1=0 ; \leftarrow \text { layer } S^{\prime} \rightarrow(00)^{\prime}\end{array}\right.$
$\operatorname{AFM}\left(\begin{array}{l}d \\ d^{\prime}\end{array}\right) \rightarrow\left\{\begin{array}{l}2 \alpha \gamma \beta \delta^{*}+(\beta-\alpha)\left(\gamma+\delta^{*}\right)=2 ; \leftarrow \text { layer } S \rightarrow(\tilde{\pi}) \\ \alpha^{\prime} \delta^{*}+1=0 ; \leftarrow \text { layer } S^{\prime} \rightarrow(\pi 0)^{\prime} .\end{array}\right.$

In this case we also have three solutions formally coinciding with ones from known classification scheme [1,31,32]: $\operatorname{AFM}\left(c^{\prime}\right)=(00), \operatorname{AFM}(c)=$ $=(0 \pi), \operatorname{AFM}\left(d^{\prime}\right)=(\pi 0)$. The $\operatorname{AFM}(d)$ solution is the new $\tilde{\pi} \pi$ one.

In the general case, there are 4 different solution sets $\operatorname{FM}\left(a, a^{\prime}\right), \operatorname{FM}\left(b, b^{\prime}\right), \operatorname{AFM}\left(c, c^{\prime}\right)$, and $\operatorname{AFM}\left(d, d^{\prime}\right)$ for the $\mathrm{S}$ and $\mathrm{S}^{\prime}$ layers, each of which completely defines the state of both layers and, hence, the corresponding reduced transition temperatures $t_{c}$ and $t_{c}^{\prime}$ (6). However, since the solution $t_{c}$ of Eq. (6) does not change when $k_{S}$ is replaced by its complex conjugate the solutions $t_{c}^{\prime}$ for the $S^{\prime}$ layer do not depend on relative orientation of the magnetizations: the solution of the equation (6) for the $\operatorname{FM}\left(a^{\prime}\right)$ case coincides with the solution for the AFM $\left(c^{\prime}\right)$ case. The same is true for the solutions for the $\operatorname{FM}\left(b^{\prime}\right)$ and $\operatorname{AFM}\left(d^{\prime}\right)$ cases. Thus, we have only two distinguishable solutions $a^{\prime}$ and $b^{\prime}$ for the $\mathrm{S}^{\prime}$ layer.

The latter can be easily understood from the physical point of view. Only one ferromagnetic layer $\left(F^{\prime}\right)$ acts on the outer $\mathrm{S}^{\prime}$ layer. As a result the state of the layer depends only on the magnitude of the exchange field in the $\mathrm{F}^{\prime}$ layer and does not depend neither on its sign nor on mutual ordering of the magnetizations. In other words, the $\mathrm{S}^{\prime}$ layer is always in the local ferromagnet (FM) environment, therefore the $\pi$ magnetic solutions do not exist for this layer.

The $\mathrm{F} / \mathrm{S} / \mathrm{F}^{\prime} / \mathrm{S}^{\prime} / \mathrm{F}^{\prime \prime}$ pentalayer (see Fig. 1,e) may have three nonequivalent configurations in which the $\mathrm{S}$ and $\mathrm{S}^{\prime}$ layers are in essentially different local environment. There are two symmetrical configurations: the first one is completely ferromagnetic (FM), when the magnetizations of all three $\mathrm{F}$ layers have the same directions (we designate this case as $\uparrow \mathrm{S} \mathrm{S}^{\prime} \uparrow$, where arrows show the direction of magnetizations $\mathbf{M}, \mathbf{M}^{\prime}$, and $\mathbf{M}^{\prime \prime}$ ); and the second case is antiferromagnetic (AFM or $\uparrow S \downarrow S^{\prime} \uparrow$, as shown in Fig. 1,e). The third case is the nonsymmetrical one ( $\left.\uparrow \mathrm{S} \mathrm{S}^{\prime} \downarrow\right)$, and for this mixed case we introduce the FMAFM designation.

In the first symmetrical cases we have two sets $\operatorname{FM}\left(a, a^{\prime}\right)$ and $\operatorname{FM}\left(b, b^{\prime}\right)$ of solutions (for the pentalayer states notation we use underlined letters). By symmetry, into each set we have the solutions coinciding for the $\mathrm{S}$ and $\mathrm{S}^{\prime}$ layers. Actually, the $\operatorname{FM}(\underline{a})=$ $=\mathrm{FM}\left(a^{\prime}\right)$ solutions are equal to the 00 one, defined by (7), and they coincide with $\operatorname{FM}(a)=\operatorname{FM}\left(a^{\prime}\right)=$ $=\operatorname{AFM}\left(c^{\prime}\right)$ solutions for the tetralayer (see Eqs. (20), (21)). In turn, the $\operatorname{FM}(\underline{b})=\operatorname{FM}\left(\underline{b}^{\prime}\right)$ solutions are equal to the $\tilde{\pi 0}$ one, and they coincide with $\mathrm{FM}(b)$ solution for the inner $\mathrm{S}$ layer of tetralayer, defined in (20).

In the second symmetrical $\uparrow \mathrm{S} \downarrow \mathrm{S}^{\prime} \uparrow$ case we also have two sets $\operatorname{AFM}\left(\underline{c}, \underline{c}^{\prime}\right)$ and $\operatorname{AFM}\left(\underline{d}, \underline{d^{\prime}}\right)$ solutions coinciding into each set for both $\mathrm{S}$ and $\mathrm{S}^{\prime}$ layers. So, the $\operatorname{AFM}(\underline{c})=\operatorname{AFM}\left(\underline{c^{\prime}}\right)$ solutions are equal to $(0 \pi)$ one, defined by (12), and they coincide with $\operatorname{AFM}(c)$ solution for the inner $\mathrm{S}$ layer of tetralayer (21). Two other solutions $\operatorname{AFM}(d)=\operatorname{AFM}\left(d^{\prime}\right)=(\tilde{\pi} \pi)=\operatorname{AFM}(d)$, the last one is defined in Eq. (21) for the inner $\mathrm{S}$ layer of tetralayer too.

At last, in the nonsymmetrical $\uparrow \mathrm{S} \mathrm{S}^{\prime} \downarrow$ case we have two sets FMAFM $\left(\underline{e}, \underline{e}^{\prime}\right)$ and FMAFM $\left(f, f^{\prime}\right)$ of nonequivalent solutions which coincide with corresponding ones for tetralayer (20), (21): 


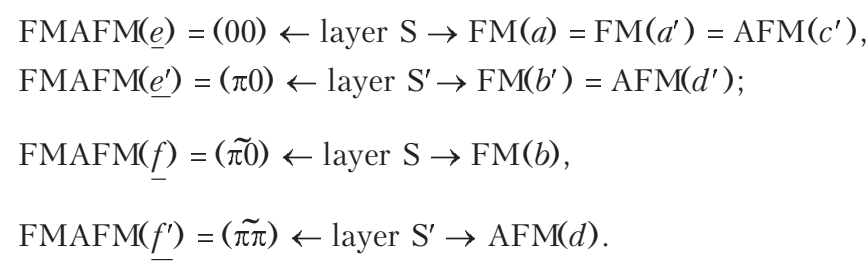

Thus, the expressions obtained above include a competition between the 0 phase and the $\pi$ phase types of superconductivity. They also take into account interaction of the localized moments of adjacent ferromagnetic layers through the superconducting layers.

In the next section we will examine the obtained solutions and clarify the winners in the interplay of the possible states.

\section{Discussion of the phase diagrams of layered F / S systems}

The set of equations (6)-(21) allows us to investigate the dependence of critical temperatures $\left(t_{c}\right.$ or $\left.t_{c}{ }_{c}\right)$ of the layered $\mathrm{F} / \mathrm{S}$ systems on the reduced thickness of the magnetic layer $d_{f} / a_{f}=\tilde{d}$.

Keeping in mind a possibility of an application of the system as a «spin device» we have searched for such a set of parameters for which the difference between the various states of studied systems is sufficiently large to be observed. After performing numerous computer experiments we have found a range for the values of the parameters that satisfies these conditions. The optimal range of parameters should be as follows: the boundary should be sufficiently transparent $\left(\sigma_{s} \geq 5 \gg 1\right)$, the ferromagnetic metal should be sufficiently dirty or (and) weak enough in regard to its magnetic properties $\left(2 I \tau_{f}=l_{f} / a_{f} \gtrsim 0.15<1\right)$, and the parameter $n_{s f}=N_{s} v_{s} / N_{f} v_{f}>1$. From experimental viewpoint this constraint on the values of the parameters does not look unreasonable.

A set of phase curves $t_{c}\left(d_{f}\right)$ for bilayer, trilayers, and superlattices for the optimal values of the parameters is shown in Fig. 2. These systems are well explored (see, for example, Ref. 1 and references therein), but this picture allows us to explain the phase diagram of all stated layered $\mathrm{F} / \mathrm{S}$ systems with unified positions and to compare them with each other and with ones for the $\mathrm{F} / \mathrm{S}$ tetra- and pentalayer (see Figs. 3, 4 below).

So, the $\mathrm{F} / \mathrm{S}$ bilayer (Fig. $2, a$ ) has only possible solution (00). Note, that critical temperature has reentrant behavior. The same type of $T_{c}\left(d_{f}\right)$ dependence was firstly predicted by us $[8,9]$, and it was observed in the $\mathrm{Fe} / \mathrm{V} / \mathrm{Fe}$ trilayer not long ago [17].

In the $\mathrm{F} / \mathrm{S} / \mathrm{F}$ trilayer (Fig. $2, b$ ) the competition of two magnetic states takes place. Due to partial compensation of paramagnetic effect of exchange field
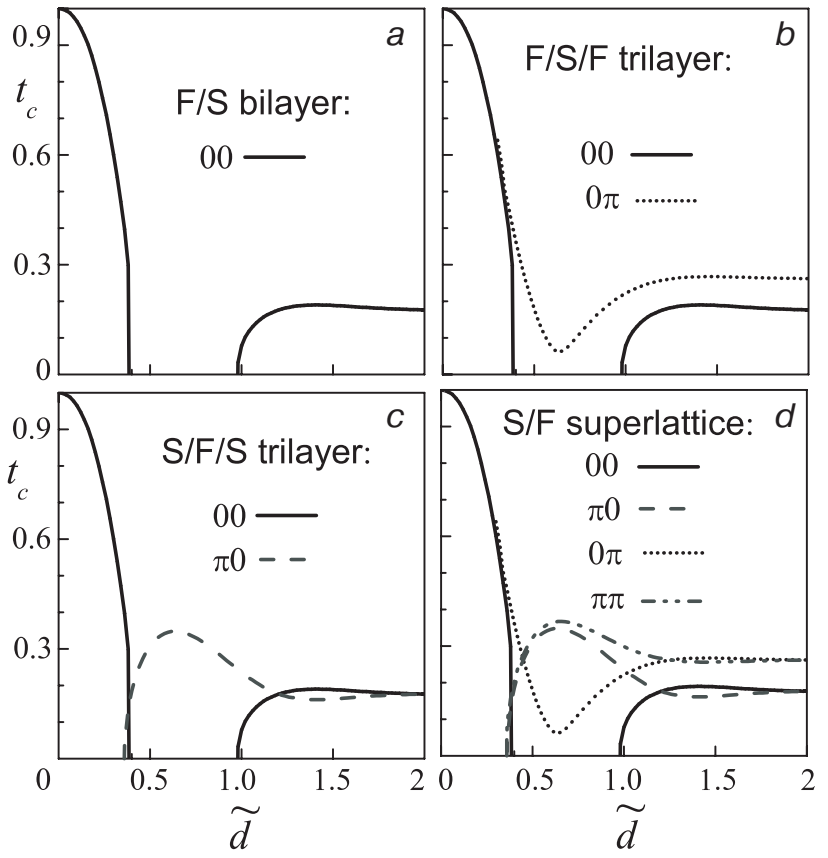

Fig. 2. The phase diagrams $\left(t_{c}-\tilde{d}\right)$ of the $\mathrm{F} / \mathrm{S}$ nanostructures for the following values of parameters: $\sigma_{s}=15,2 I \tau_{f}=0.1, n_{s f}=1.4, l_{s}=0.25 \xi_{s 0}$, and $d_{s}=0.72 \xi_{s 0}$. In the figure $t_{c}=T / T_{C S}$ is the reduced temperature, and $\tilde{d}=d_{f} / a_{f}$ is the reduced $\mathrm{F}$ layer thickness. The phase diagram of the $\mathrm{F} / \mathrm{S}$ bilayer with one possible state (00) (a); the phase diagram of the $\mathrm{F} / \mathrm{S} / \mathrm{F}$ trilayer for the $\mathrm{FM}$ (00) and $\operatorname{AFM}(0 \pi)$ configurations $(b)$; the phase diagram of the $\mathrm{S} / \mathrm{F} / \mathrm{S}$ trilayer with competition between 0 phase and $\pi$ phase of superconductivity $(c)$; the phase diagram of the $\mathrm{F} / \mathrm{S}$ superlattice where the thicknesses of all $\mathrm{F}$ layers equal $d_{f}$, and the thicknesses of all $\mathrm{S}$ layers equal $d_{s}(d)$.

$I$ the $\pi$ magnetic state $(0 \pi)$ has higher critical temperature than the 00 state. Therefore this $0 \pi$ state wins and, according to the theory of second order phase transitions, the $\mathrm{F} / \mathrm{S} / \mathrm{F}$ trilayer always is in AFS condition at $T \leq T_{c}(0 \pi)$. But if we turn on a weak external magnetic field $H$ greater than coercive field, $H_{\text {coer }}$, we can go the trilayer into the FM state in which the magnetizations of both $\mathrm{F}$ layers are parallel, and the system becomes normal. As noted earlier $[22,23]$, this system could be used for superconducting «spin switch» construction with two possible states.

As to the $\mathrm{S} / \mathrm{F} / \mathrm{S}$ trilayer (Fig. 2,c), the spontaneous transitions take place with increasing thickness of the $\mathrm{F}$ layer. The first one is from the 0 superconducting state (00), when both $S$ layers have the same sign of superconducting order parameter $\Delta$, to the $\pi$ superconducting state state $(\pi 0)$, when $\Delta$ in the S layers $\underset{\sim}{\operatorname{differ}}$ by sign, at $\tilde{d} \simeq 0.4$. The reverse transition is at $\tilde{d} \simeq 1.2$. The such trilayers are often used for experimental investigation of so called the $\pi$ junctions by 
the Josephson current methods (see, for example, Ref. 2).

At last we see all four states in the phase diagram for the $\mathrm{F} / \mathrm{S}$ superlattice (Fig. 2,d) for which, properly speaking, the classification scheme has been proposed $[1,31,32]$. In cited references the detail discussion of the properties of such multilayered system connected with competition between the 0 and $\pi$ phase superconductivity and the 0 and $\pi$ phase magnetism can be found. Emphasize, that in the AFM condition due to partial compensation of paramagnetic effect of exchange field the $\mathrm{F} / \mathrm{S}$ superlattice has the higher $T_{c}$ than one in the FM state. The complicated phase diagram of the $\mathrm{F} / \mathrm{S}$ superlattice allowed us to propose a spin device with five possible states controlled by small external magnetic field [35].

Completing the short review of phase diagram of studied earlier $\mathrm{F} / \mathrm{S}$ system, we stress that all layered $\mathrm{F} / \mathrm{S}$ structures presented in Fig. 2 posses single critical temperature $T_{c}$ for all $\mathrm{S}$ layers.

\subsection{Decoupled superconductivity in the $\mathrm{F} / \mathrm{S}$ tetra- and pentalayer}

The set of phase diagram for the $\mathrm{F} / \mathrm{S} / \mathrm{F}^{\prime} / \mathrm{S}^{\prime}$ tetralayer is presented in Fig. 3.

As one might expect, the $a^{\prime}$ and $b^{\prime}$ curves for the $\mathrm{S}^{\prime}$ layer in Figs. 3, $a$ and 3,b are identical for both FM and AFM configurations, and the $a^{\prime}$ curve for the $\mathrm{S}^{\prime}$ layer coincides completely with the $a$ curve for the $\mathrm{S}$ layer since both of them describe the same 00 state. The rest of the states for the inner S layer (the $b$ curve for the FM configuration, and the $c$ and $d$ curves for the AFM one) have different dependencies as compared with the ones for the $\mathrm{S}^{\prime}$ layer and each other.

Hence as it follows from Figs. 2, 3 and discussion presented here the tetralayer has more physically different states than the $\mathrm{F} / \mathrm{S} / \mathrm{F}$ trilayer and even the $\mathrm{F} / \mathrm{S}$ superlattice!

Thus, the $00, \pi 0$, and $0 \pi$ states of the four-layered system (Figs. 3, $a, b$ ) correspond to the same states of the superlattice (Fig. 3,c). The $\tilde{\pi 0}$ state (the $b$ curve in Fig. $3, a$ ) and the $\tilde{\pi} \pi$ one (the $d$ curve in Fig. $3, b$ ) are the states associated with the $\pi$ phase superconductivity. These are the new solutions which are not found in the superlattice case (Fig. 2, $d$ ). The main difference between the new $\tilde{\pi} \tilde{x}$ and the known $\pi \chi$ states is the peak position. For the inner $\mathrm{S}$ layer it is shifted to the lower values of the $d_{f}$ thickness compared with the superlattice case due to the implementation of the external boundary conditions (3b).

The above-mentioned peculiarities of the four-layered system lead to different critical temperatures of different $\mathrm{S}$ layers. To show this consider the FM configuration (Fig. 3,a) in detail. If there is no difference
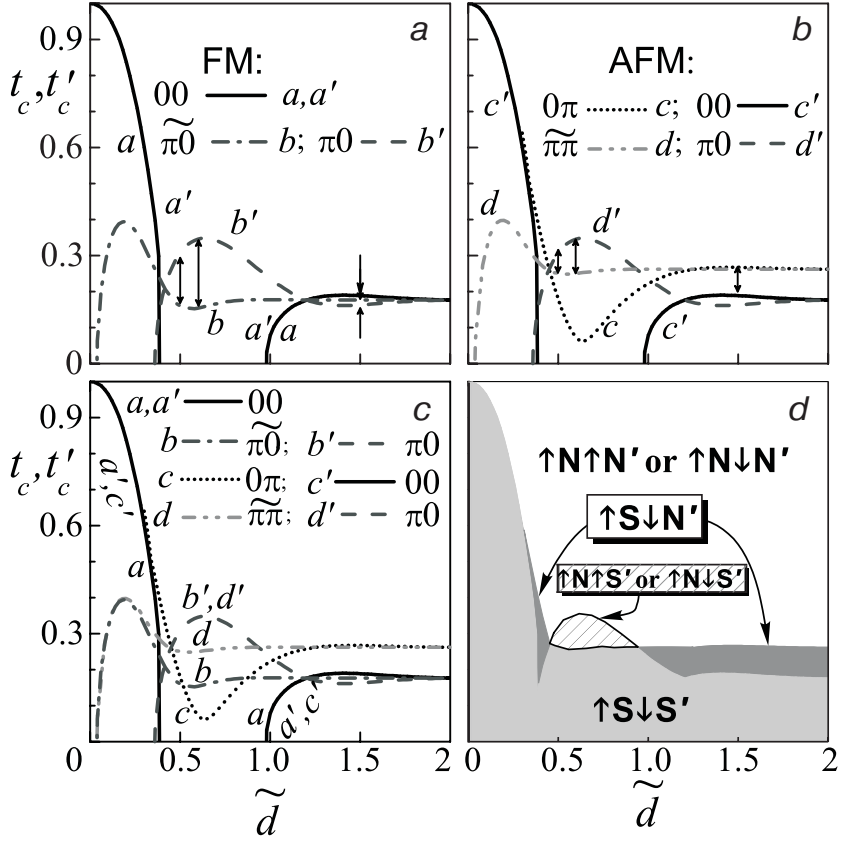

Fig. 3. The phase diagrams $\left(t_{c}-\tilde{d}\right)$ of the $\mathrm{F} / \mathrm{S} / \mathrm{F}^{\prime} / \mathrm{S}^{\prime}$ tetralayer for the same set of parameters as in Fig. 2. The $t_{c}^{\prime}$ curves for the outer $\mathrm{S}^{\prime}$ layer are denoted using letters with a prime. The letters without a prime indicate the $t_{c}$ curves for the inner S layer. All denotations of curves correspond to ones entered in Eqs. (20) and (21). In the panels $a$ and $b$ the arrows show the $\left(t_{c}-t_{c}^{\prime}\right)$ difference between the states which are discussed in the paper. The phase diagram for the FM configuration of the magnetizations of both $\mathrm{F}$ layers $(a)$; the phase diagram for the AFM configuration $(b)$; the combined phase diagram of the $\mathrm{F} / \mathrm{S} / \mathrm{F}^{\prime} / \mathrm{S}^{\prime}$ tetralayer $(c)$; the generalized phase diagram of the tetralayer $(d)$. Vertical arrows show the direction of the magnetization in the corresponding ferromagnetic layer. The letters $\mathrm{S}$ and $\mathrm{N}$ stand for the superconducting and normal states of the superconducting layers, respectively.

between $t_{c}$ and $t_{c}^{\prime}$ for the 00 state then the case of the $\pi$ phase superconductivity is more interesting since there is a difference between $t_{c}^{\prime}(\pi 0)$ and $t_{c}(\tilde{\pi} 0)$. Actually for each superconducting layer the upper envelope curve is realized due to free energy minimum condition. In the case of the FM configuration that will be $a^{\prime}-b^{\prime}-a^{\prime}$ curve and $a-b-a$ one for the $\mathrm{S}^{\prime}$ and $\mathrm{S}$ layers, respectively. This leads to switching the ground state between the states with the 0 and $\pi$ superconducting phases as the thickness $\tilde{d}$ changes just as it was shown for the $\mathrm{S} / \mathrm{F} / \mathrm{S}$ layer in Fig. 2,c (at $\tilde{d} \simeq 0.4$ and $\tilde{d} \simeq 1.2$, respectively).

In the $\pi$ phase superconductivity case, the order parameter $\Delta$ has opposite signs for the $S$ and $S^{\prime}$ layers. Accordingly, the pair amplitude in the inner $F^{\prime}$ layer (Eq. $(17 \mathrm{c}))$ has a sine-like behavior $\left(B^{\prime}=0\right)$ and is 
antisymmetric with respect to the layer center at which the sign change of the pair amplitude takes place while traversing the $\mathrm{F}^{\prime}$ layer. The above-mentioned different $T_{c}$ behavior in the $\mathrm{S}$ and $\mathrm{S}^{\prime}$ layers (the $b$ and $b^{\prime}$ curves in Fig. 3, $a$, respectively) leads to a difference between critical temperatures $t_{c}$ and $t_{c}^{\prime}$. For instance, at $\tilde{d}=1.5$ the reduced critical temperature of the $\mathrm{S}$ layer $t_{c}$ is equal to 0.177 , and $t_{c}^{\prime}=0.163$, at $\tilde{d}=0.5$ the difference is larger: $t_{c}^{\prime}=0.308$ and $t_{c}=0.16$. If the reduced thickness $\tilde{d}$ were equal 0.6 , the difference would be almost maximal: $t_{c}^{\prime}=0.346$, and $t_{c}=0.154$.

The reduced critical temperatures $t_{c}$ and $t^{\prime}{ }_{c}$ that correspond to these three values of the reduced thickness $\tilde{d}$ are shown in Fig. 3, $a$ by arrows. The difference between two critical temperatures $t_{c}$ and $t_{c}^{\prime}$ should be observed in experiments with the special field-cooled samples prepared with the FM ordering of the magnetizations (see Ref. 33, for experimental details).

The appearance of the critical temperature difference for the $\mathrm{F} / \mathrm{S}$ tetralayer is a manifestation of the critical temperatures hierarchy in the clearest form. The origin of the $T_{c}$ difference is obvious because, firstly, the $S$ and $S^{\prime}$ layers are situated in different magnetic environment and, secondly, they have different boundary conditions. In particular it is expressed in the above mentioned shift of peak of the new $\tilde{\pi 0}$ dependence due to the outside boundary conditions. Note, that for the same reason the critical fields $H_{c}$ and $H^{\prime}{ }_{c}$ will differ for layers $\mathrm{S}$ and $\mathrm{S}^{\prime}$, correspondingly.

For the AFM configuration of the $\mathrm{F} / \mathrm{S} / \mathrm{F}^{\prime} / \mathrm{S}^{\prime}$ system we have a similar picture (Fig. 3,b), but in this case there are four different curves. Note that all above mentioned peculiarities take place as well. As it has been discussed above, the phase curves for the $S^{\prime}$ layer are the same for both the FM and the AFM orientations. Two different solutions are obtained for the inner S layer. One of them is the known «superlattice» solution $0 \pi$ (curve $c$ ) while the second one is the new $\tilde{\pi} \pi$ solution (curve $d$ ). There is also a competition between the 0 and $\pi$ phase superconductivity that leads to a appearance of the corresponding envelope curves of the second order phase transition for the $\mathrm{S}$ and $S^{\prime}$ layers ( $c-d-c$ and $a^{\prime}-b^{\prime}-a^{\prime}$, respectively).

The $0 \pi$ solution corresponds to the $D^{\prime}=0$ and $B^{\prime} \neq 0$ case, and the $A$ and $C$ factors are not equal 0 , i.e., the pair amplitude in the $\mathrm{S}$ layer does not possess any parity. The admixture of the sine solutions to the cosine ones in expression (17b) reflects the partial compensation of the paramagnetic effect of exchange field $I$ for the $\mathrm{S}$ layer in the AFM state with antiparallel alignment of the $\mathrm{F}$ layers magnetizations. The previous statement applies to the $\tilde{\pi} \pi$ state in the $S^{\prime}$ layer too.
As in stated above FM case, the difference between $t_{c}$ and $t_{c}^{\prime}$ can be observed in experiments with the special field-cooled AFM samples.

Let us take up the common case, when there is the interplay of all the four states (20), (21), to clarify the winners in this competition. For convenience all the phase curves are shown in Figs. 3, $a, b$ in one combined diagram (Fig. 3,c).

Note, that at $\tilde{d}=0.5$ the $\tilde{\pi} \pi$ state (the $d$ curve in Fig. 3,b and in Fig. 3,c) has the highest $T_{c}$ among all possible states for the $\mathrm{S}$ layer $t_{c} \simeq 0.25$, but that is lower than the appropriate temperature for the $\pi 0$ state of the $S^{\prime}$ layer $t_{c}^{\prime} \simeq 0.31$. According to the theory of second-order phase transitions, the state possessing the lower free energy (higher $T_{c}$ ) is realized. Thus for the samples with the reduced thickness $\tilde{d}=0.5$ the $\mathrm{S}$ and $\mathrm{S}^{\prime}$ layers are both in the normal $(\mathrm{N})$ state if the reduced temperature $t>t_{c}^{\prime} \simeq 0.31$. Below $t_{c}^{\prime}$ the $\mathrm{S}^{\prime}$ layer becomes superconducting (S) but the $\mathrm{S}$ layer remains in the $\mathrm{N}$ state while $t>t_{c} \simeq 0.25$. Finally, at $t<t_{c}$ the $\operatorname{AFMS}$ state $\left(\operatorname{AFM}\left(d, d^{\prime} \equiv b^{\prime}\right)\right)$ wins and for the whole system we have the case with the $\pi$ phase superconductivity and the $\pi$ phase magnetism.

At $\tilde{d}=1.5$ we have the following chain of the second order phase transitions: $\uparrow \mathrm{N} \downarrow \mathrm{N}$ (or $\uparrow \mathrm{N} \uparrow \mathrm{N}$ ) $\stackrel{t_{c} \simeq 0.27}{\longrightarrow} \uparrow \mathrm{S} \downarrow \mathrm{N} \longrightarrow \stackrel{t_{c} \simeq 0.19}{\longrightarrow} \uparrow \mathrm{S} \downarrow \mathrm{S}$ (see a caption to Fig. 3, $d$ for an explanation of the notation). Thus at low temperatures the AFMS state $\left(\operatorname{AFM}\left(c, c^{\prime} \equiv a^{\prime}\right)\right)$ wins too, but this state is associated with the 0 phase superconductivity. Note in the framework of our theory only transition temperatures can be found and it is not possible to determine what state inside the «normal» state region is preferable.

The analogous analysis can be carried out for the entire range of the reduced $F$ layer thicknesses $(0<\tilde{d}<2)$. Assume the system can choose its own state according to the theory of the second order phase transitions. The state with higher critical temperature wins, and one of four states defined by Eqs. (20), (21) (see also Figs. 3, $a, b, c$ ) is realized for the system. A complete phase diagram constructed for the system is presented in Fig. 3, $d$. Four different regions can be defined for this diagram: at high temperatures both $\mathrm{S}$ and $S^{\prime}$ layers are in normal state and the mutual ordering of the magnetizations in the $\mathrm{F}$ and $\mathrm{F}^{\prime}$ layers is unimportant. As it follows from the phase diagram, there are two regions marked in dark grey color with $d e$ coupled superconductivity $\}$ in antiferromagnetic state for which the inner S layer is superconducting (S), and the outer $\mathrm{S}^{\prime}$ one is normal $(\mathrm{N})$. The striped marked region also corresponds to the state with decoupled superconductivity when the outer $\mathrm{S}^{\prime}$ layer is superconducting and the inner S layer is normal. Finally, at low temperatures and/or at small $d_{f}$ thicknesses the 
system is in the ground AFMS state (grey marked region).

Thus, we can predict, there are distinct regions of parameters when the $\mathrm{F} / \mathrm{S} / \mathrm{F}^{\prime} / \mathrm{S}^{\prime}$ tetralayer should be in the state with decoupled superconductivity. Moreover, if the inner $\mathrm{S}$ layer is in the superconductive state then ordering of the magnetizations should be antiferromagnetic. This is the result of the inverse action of superconductivity on magnetism.

At last let us consider the $\mathrm{F} / \mathrm{S}$ pentalayer case shown in Fig. 4. Note that the tetralayer case more comprehensively since it has the extra $\pi 0$ state in comparison with the pentalayer one. This leads to that the $\mathrm{F} / \mathrm{S}$ pentalayer possesses simpler phase diagrams. Naturally, in the symmetrical cases the both $\mathrm{S}$ and $\mathrm{S}^{\prime}$ layers have identical critical temperatures $T_{c}=T_{c}^{\prime}$.

In the FM case (Fig. 4, $a$ ) the both $\mathrm{S}$ layers may be simultaneously either in the 00 state (curve $\underline{a}=\underline{a}^{\prime}$ ) or in the $\pi 0$ state (curve $b=b^{\prime}$ ), that correspond to the $\left(a, a^{\prime}\right)$ and $b^{\prime}$ curves for the tetralayer case in Fig. 4,a, respectively. Competition between the 0 and $\pi$ phase superconductivity leads to switching ground state as it was shown in the tetralayer case above.

In the AFM case (Fig. 4,b), by symmetry, we have single $T_{c}$ for all system too, and the states of the both $\mathrm{S}$ layers are also determined simultaneously either the $0 \pi$ one (curve $c=\underline{c}^{\prime}$ ) or the $\tilde{\pi} \pi$ one (curve $d=d^{\prime}$ ). These states correspond to the $c$ and $d$ curves for the inner $\mathrm{S}$ layer in Fig. 3,b for the tetralayer, accordingly.

In the nonsymmetrical FMAFM case each of the superconducting layers is situated in different local magnetic surroundings, and we have a competition between the $\left(\underline{e}, \underline{e}^{\prime}\right)$ set and $\left(f, f^{\prime}\right)$ set (see Fig. $\left.4, c\right)$. The 0 phase superconducting state $\left(\underline{e}, \underline{e^{\prime}}\right)$ wins at $\tilde{d} \lesssim 0.4$ and $\tilde{d} \gtrsim 1.2$. The $\pi$ phase superconducting state $\left(f, f^{\prime}\right)$ is energetically preferable at $0.4 \lesssim \tilde{d} \lesssim 1.2$ conditions. In any case the critical temperature $T^{\prime}{ }_{c}$ of layer $\mathrm{S}^{\prime}$ situated in the local AFM surroundings is higher than temperature $T_{c}$ of layer $\mathrm{S}$ situated in the local FM surroundings. It is physically transparently because the partial compensation of paramagnetic effect of exchange field $I$ takes place only for the $S^{\prime}$ layer.

Thus in only such nonsymmetrical case the decoupled superconductivity and the $T_{c}$ difference are possible for the $\mathrm{F} / \mathrm{S}$ pentalayer. This effect could be observed in special prepared samples in field-cooled FMAFM configuration.

In common case this FMAFM configuration is energetically less favorable than symmetrical AFM case, so that this pentalayer can have only AFMS state (see Fig. 4, $d$ ). Comparing corresponding diagrams for the tetralayer (Fig. 3) and pentalayer (Fig. 4) cases we conclude that the $\mathrm{F} / \mathrm{S} / \mathrm{F}^{\prime} / \mathrm{S}^{\prime}$ tetralayer has more rich

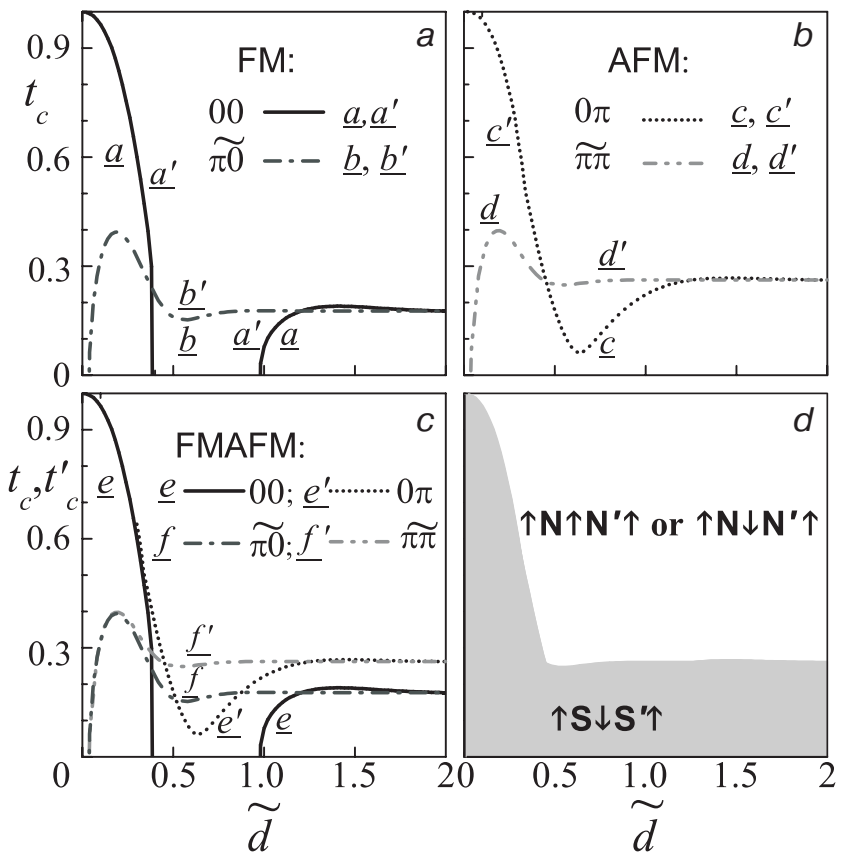

Fig. 4. The phase diagrams of the $\mathrm{F} / \mathrm{S} / \mathrm{F}^{\prime} / \mathrm{S}^{\prime} / \mathrm{F}^{\prime \prime}$ pentalayer with the same parameters as in Figs. 2, 3. All denotations of curves and states correspond to ones entered in the last part of Sec. 2. The phase diagram for the FM configuration of the magnetizations of all $\mathrm{F}$ layers (a); the phase diagram for the AFM configuration (b); the phase diagram for the FMAFM configuration $(c)$; the generalized phase diagram of the pentalayer (the designations follow to ones used in Fig. 3,d) $(d)$.

properties in physical sense. It proves that this $\mathrm{F} / \mathrm{S}$ system is the most perspective candidate for using in the superconducting nanoelectronics.

Note in conclusion, the details of the phase diagram significantly depend on the choice of the system parameters and the above analysis was carried out assuming the absence of an external magnetic field $(H=0)$.

\section{Conclusions}

The few-layered $\mathrm{F} / \mathrm{S}$ systems have been consistently studied within the modern theory of the proximity effect with a detailed account of the boundary conditions given. Theoretical studies of the critical temperature dependence on the thicknesses of the $\mathrm{F}$ layers have been performed as well for a wide range of parameters, and a physically interesting range of their values has been determined. The latter should be of help in choosing materials and technology for preparation of the $\mathrm{F} / \mathrm{S}$ systems with predetermined properties.

It has been shown that when the $\pi$ phase superconductivity coexists with the nonequivalence of all lay- 
ers the physics of the $\mathrm{F} / \mathrm{S}$ tetralayer is considerably richer in comparison with one for the earlier studied $\mathrm{F} / \mathrm{S} / \mathrm{F}$ trilayer $[22,23]$, the $\mathrm{F} / \mathrm{S}$ superlattices $[1,31,32]$, and even the $\mathrm{F} / \mathrm{S}$ pentalayer studied here. For tetra- and pentalayer the new $\pi$ phase superconducting states have been found. The hierarchy of critical temperatures is manifested mainly through the occurrence of the different critical temperatures in the different $S$ and $S^{\prime}$ layers (decoupled superconductivity). This prediction can be experimentally verified both for the common case and for the specially prepared field-cooled samples.

Theoretical studies performed in this paper have shown that the four-layered $\mathrm{F} / \mathrm{S} / \mathrm{F}^{\prime} / \mathrm{S}^{\prime}$ system has the best prospects for its use in superconducting spin electronics (superconducting spintronics). This system can be used for a creation of an essentially new type of nanoelectronics combining within the same layered sample the advantages of the superconducting and magnetic channels of data recording that are associated with the conducting properties of both $\mathrm{S}$ and $\mathrm{S}^{\prime}$ layers and the magnetic ordering of the magnetizations of the ferromagnetic layers as it is shown for the $\mathrm{F} / \mathrm{S}$ superlattice case $[1,35]$. Preliminary estimates show that the possible spin device based on the studied $\mathrm{F} / \mathrm{S}$ tetralayer can have up to seven different states, and transitions between these states can be controlled by a external magnetic field. Certainly, low temperatures at which usual «cold» superconductivity is possible would be a condition for the use of this type of control device. However, similar superconducting devices on the basis of the $\mathrm{F} / \mathrm{S}$ structures with $\mathrm{S}$ layers made out of high-temperature superconducting materials [36] should work at much higher temperatures.

Y.P. and M.K. are grateful to the Max-Planck-Institute for the Physics of Complex Systems (Dresden) for providing excellent conditions for fruitful work. Y.P. thanks Prof. Fulde and the participants of his seminar for the productive discussion of the results presented in this paper. This work was supported in part by RFBR (No 04-02-16761, No 05-02-16369).

1. Y.A. Izyumov, Y.N. Proshin, and M.G. Khusainov, Usp. Fiz. Nauk 172, 113 (2002); Physics-Uspekhi 45, 109 (2002).

2. V.V. Ryazanov, V.A. Oboznov, A.S. Procofiev, V.V. Bolginov, and A.K. Feofanov, J. Low Temp. Phys. 136, 385 (2004).

3. A.A. Golubov, M.Y. Kupriyanov, and E. Il'ichev, Rev. Mod. Phys. 76, 411 (2004).

4. A. Buzdin, Rev. Mod. Phys. 77, 935 (2005).

5. A.I. Buzdin, B. Vujićić, and M.Y. Kupriyanov, Zh. Eksp. Teor. Fiz. 101, 231 (1992) [Sov. Phys. JETP 74, 124 (1992)].
6. Z. Radović, M. Ledvij, L. Dobrosavljević-Grujić, A.I. Buzdin, and J.R. Clem, Phys. Rev. B44, 759 (1991).

7. L.N. Bulaevskii, V. Kuzii, and A. Sobyanin, Pis'ma Zh. Eksp. Teor. Fiz. 25, 314 (1977) [JETP Lett. 25 290 (1977)].

8. M.G. Khusainov and Y.N. Proshin, Phys. Rev. B56, 14283 (1997); see also Phys. Rev. B62, 6832 (2000).

9. Y.N. Proshin and M.G. Khusainov, Pis'ma Zh. Eksp. Teor. Fiz. 66, 527 (1997) [JETP Lett. 66, 562 (1997)].

10. Y.N. Proshin and M.G. Khusainov, Zh. Eksp. Teor. Fiz. 113, 1708 (1998); see also Zh. Eksp. Teor. Fiz. 116, 1882 (1999); JETP 86, 930 (1998); ibid. 89, 1021 (1999).

11. J. Aarts, J.M. E. Geers, E. Brück, A.A. Golubov, and R. Coehoorn, Phys. Rev. B56, 2779 (1997).

12. L.R. Tagirov, Physica C307, 145 (1998).

13. Y.V. Fominov, N.M. Chtchelkatchev, and A.A. Golubov, Pis'ma Zh. Eksp. Teor. Fiz. 74, 101 (2001) [JETP Lett. 74, 96 (2001)].

14. Y.V. Fominov, N.M. Chtchelkatchev, and A.A. Golubov, Phys. Rev. B66, 014507 (2002).

15. A. Bagrets, C. Lacroix, and A. Vedyayev, Phys. Rev. B68, 054532 (2003).

16. Y.A. Izyumov, Y.N. Proshin, and M.G. Khusainov, Pis'ma Zh. Eksp. Teor. Fiz. 71, 202 (2000) [JETP Lett. 71, 138 (2000)].

17. I.A. Garifullin, D.A. Tikhonov, N.N. Garif'yanov, L. Lazar, Y.V. Goryunov, S.Y. Khlebnikov, L.R. Tagirov, K. Westerholt, and H. Zabel, Phys. Rev. B66, 020505 (2002).

18. A.I. Larkin and Y.N. Ovchinnikov, Zh. Eksp. Teor. Fiz. 47, 1136 (1964) [Sov. Phys. JETP 20, 762 (1965)].

19. P. Fulde and R.A. Ferrell, Phys. Rev. A135, 550 (1964).

20. E.A. Demler, G.B. Arnold, and M.R. Beasley, Phys. Rev. B55, 15174 (1997).

21. M.G. Khusainov and Y.N. Proshin, Usp. Fiz. Nauk 173, 1385 (2003) [Phys. Usp. 46, 1311 (2003)].

22. A.I. Buzdin, A.V. Vedyayev, and N.V. Ryzhanova, Europhys. Lett. 48, 686 (1999).

23. L.R. Tagirov, Phys. Rev. Lett. 83, 2058 (1999).

24. F.S. Bergeret, K.B. Efetov, and A.I. Larkin, Phys. Rev. B62, 11872 (2000).

25. F.S. Bergeret, A.F. Volkov, and K.B. Efetov, Phys. Rev. B69, 174504 (2004).

26. V. Krivoruchko and V. Varyukhin, Ukr. J. Phys. 50, 795 (2005).

27. F.S. Bergeret, A.F. Volkov, and K.B. Efetov, Phys. Rev. Lett. 86, 4096 (2001).

28. F.S. Bergeret, A.F. Volkov, and K.B. Efetov, Phys. Rev. B68, 064513 (2003).

29. A.F. Volkov, F.S. Bergeret, and K.B. Efetov, Phys. Rev. Lett. 90, 117006 (2003).

30. Y.V. Fominov, A.A. Golubov, and M.Y. Kupriyanov, Pis'ma Zh. Eksp. Teor. Fiz. 77, 609 (2003) [JETP Lett. 77, 510 (2003). 
31. M.G. Khusainov, Y.A. Izyumov, and Y.N. Proshin, Pis'ma Zh. Eksp. Teor. Fiz. 73, 386 (2001) [JETP Letters 73, 344 (2001).

32. Y.N. Proshin, Y.A. Izyumov, and M.G. Khusainov, Phys. Rev. B64, 064522 (2001).

33. J.P. Goff, P.P. Deen, R.C. C. Ward, M.R. Wells, S. Langridge, R. Dalgleish, S. Foster, and S. Gordeev, JMMM 240, 592 (2002).
34. S. Oh, D. Youm, and M.R. Beasley, Appl. Phys. Lett. 71, 2376 (1997)

35. Y. Proshin, Y. Izyumov, and M.G. Khusainov, Supercond. Sci. Techn. 15, 285 (2002).

36. H.-U. Habermeier and G. Cristiani, Phys. Status Solidi A201, 1436 (2004). 